\title{
Imagination et mobilité esthétique
}

\author{
Par PATRICIA LIMIDO
}

Université de Rennes 2

Résumé L'expérience esthétique est pensée depuis Kant comme relevant d'une attitude désintéressée, essentiellement fondée sur un jeu de l'imagination. Mais pour désintéressée et déréalisante qu'elle soit, cette attitude est aussi créatrice d'œuvres, de plaisirs et d'évaluations réelles, et si elle s'exerce de manière privilégiée dans le monde des œuvres de l'art, elle trouve aussi une impulsion légitime dans la perception de la nature, des choses et des paysages naturels. Il conviendra alors de se demander si, dans ce dernier cas, la mise entre parenthèses des positions naturelles est encore compatible avec l'appréhension de ce qui est justement nature ? Autrement dit, si une expérience esthétique de la nature est possible, quelle part l'imagination y joue-t-elle et si dans cette expérience, l'imagination schématise sans concept, jusqu'où la connaissance peut-elle néanmoins y prendre part? Il s'agira dès lors de revisiter l'expérience esthétique de la nature et pour cela de revenir sur le régime intentionnel des facultés qui s'y exercent afin de confronter les jeux de l'imagination aux données sensorielles et aux savoirs des sciences naturelles, afin aussi d'interroger la nature du plaisir esthétique entre sensualité et réflexion.

\section{Introduction}

Si l'imagination ouvre un espace de neutralité qui rend possible l'attitude esthétique, elle favorise aussi la naissance d'un plaisir spécifique qui, pour sa part, n'offre pas les caractères du modifié mais s'affirme bien comme « un se-réjouir actuel » ${ }^{1}$, selon le mot de Husserl. Par là-même, le jeu de l'imagi-

${ }^{1}$ E. Husserl, Husserliana XXIII, texte $\mathrm{n}^{\circ} 15$, Mode de reproduction et phantasia. Conscience d'image (également en relation avec les prises d'attitude) mars-avril 
nation esthétique nous incline à jeter un pont entre le modifié et l'actuel, à instaurer un espace de flottement et d'aller-retour entre le réel et l'imaginé. De même la production des Idées esthétiques dans le jeu créatif et leur réception chez le spectateur supposent des échanges troubles entre réel et irréel, subjectif et objectif, sensible et intelligible.

Mais l'attitude esthétique, pour désintéressée et déréalisante qu'elle soit, peut aussi trouver son impulsion légitime dans la perception de la nature, de choses ou de paysages naturels. Il conviendra alors de se demander si, dans ce dernier cas, la mise entre parenthèses des positions naturelles est encore compatible avec l'appréhension de ce qui est justement " nature »? Autrement dit, est-ce qu'en ce cas, la réduction n'est pas vouée à être seulement amorcée, puis désamorcée pour s'ouvrir à la délectation du sensible naturel ?

Cela pose deux problèmes. Tout d'abord celui de déterminer en quoi cette expérience est encore esthétique et non pas seulement sensorielle. Ensuite, celui de savoir si l'appréciation esthétique de la nature suppose une connaissance (quelconque ou avancée) de la nature: car de même que l'appréciation esthétique de l'œuvre d'art suppose le " goût», soit un certain savoir de la culture artistique, de même peut-être l'appréciation de la nature suppose-t-elle un savoir physique des phénomènes naturels. Tel est le postulat de l'esthétique environnementale défendue, par exemple, par l'américain Allen Carlson et qu'il s'agira aussi d'interroger car s'il ne va pas de soi que la contemplation de la nature se fasse sans aucun savoir, il ne va pas non plus de soi que ce savoir en conditionne l'évaluation esthétique.

C'est pourquoi il me paraît utile de revisiter l'expérience esthétique de la nature afin d'y confronter les jeux de l'imagination aux données sensorielles et aux savoirs des sciences naturelles, afin aussi d'interroger la nature du plaisir esthétique entre sensualité et intellection. Je m'appuierai ici principalement sur l'esthétique kantienne autant que sur les analyses esthétiques développées par Husserl, en particulier dans le volume XXIII des Husserliana: Phantasia, conscience d'image, souvenir. La continuité des deux approches demanderait bien sûr à être argumentée mais ce ne sera pas mon propos ici, et je renvoie aux travaux qui s'y sont déjà confrontés ${ }^{1}$. Je m'en

1912, p. [393], trad. fr : Phantasia, Conscience d'image, Souvenir, Grenoble, Jérôme Millon, 2002, p. 379.

${ }^{1}$ Je renvoie sur ce point aux travaux de Danielle Lories, « Remarks on Aesthetic Intentionality: Husserl or Kant », International Journal of Philosophical Studies, vol. 14 (1), 2006, p. 31-49; on peut également consulter Paul Crowther, The Kantian 
tiendrai au fait que l'exigence de mettre l'existence entre parenthèses installe la démarche kantienne dans une dimension au moins pré-phénoménologique. Je commencerai par quelques rappels des grands principes de l'esthétique kantienne qui nous serviront de fil conducteur.

\section{L'attitude esthétique entre désintéressement et déréalisation}

La nécessité ${ }^{1}$ de fonder le jugement de goût conduit Kant à une clarification de la forme de l'expérience esthétique pure — l'expérience du beau — par opposition à l'expérience sensorielle de l'agréable. Cette clarification dévoile de manière puissante le rôle moteur et mobile de l'imagination dans la vie de la pensée.

En distinguant le beau et l'agréable, Kant établit la dimension singulière de l'expérience esthétique en tant qu'expérience désintéressée, c'est-à-dire délestée de tout intérêt théorique, moral ou économique. On a souvent souligné le caractère paradoxal voire improbable de ce désintéressement. Pourtant, même des analyticiens américains qui, en général, ne sont pas favorables à l'esthétique kantienne, ont su préciser le sens de ce désintérêt. Ainsi Jerome Stolnitz fait la liste des intérêts les plus courants qui, justement, doivent ici être mis entre parenthèses ou suspendus, et il insiste sur le fait que l'attention à l'œuvre n'est pas guidée par un intérêt cognitif, c'est-à-dire un intérêt "pris à acquérir une connaissance ${ }^{2}$. Il l'illustre ainsi : «Un météorologiste se soucie, non pas de l'apparence visuelle d'une formation saisissante de nuages, mais des causes qui l'ont amenée. De même, l'intérêt que le sociologue ou l'historien manifeste pour une ouvre d'art est cognitif ». Et dans le même rejet, il ajoute que le critique d'art n'est pas non plus dans une attitude esthétique, car sa perception de l'objet est motivée par la volonté de produire un jugement, une comparaison ou une estimation ${ }^{3}$.

Aesthetics. From Knowledge to the Avant-Garde, Oxford: Oxford University Press, 2010.

${ }^{1}$ Afin de comprendre l'unité des trois facultés de l'esprit, et d'envisager une liaison entre la faculté de connaître, légiférant dans le domaine de la nature, et la faculté de désirer œuvrant dans le domaine de la liberté, soit un passage entre l'entendement et la raison grâce à la faculté de juger (E. Kant, Critique de la faculté de juger, Paris, Vrin, 1984, Introd., IV, p. 27).

2 J. Stolnitz, «L'attitude esthétique », dans D. Lories (dir.), Philosophie analytique et esthétique, Paris, Klincksieck, 2004, p. 103-114.

${ }^{3}$ Chez Kant (Critique de la faculté de juger, §34), la critique d'art est ramenée à un art qui recherche des règles physiologiques ou psychologiques (donc empiriques) 
Ces dernières remarques sont essentielles à double titre. D'une part, elles permettent de saisir la forme spécifique de l'expérience esthétique en la démarquant des autres expériences intellectuelles que nous faisons habituellement. D'autre part, en nous obligeant à penser notre rapport à un objet autrement que sous un angle cognitif, ou intellectuel en un sens large, elles permettent aussi de ramener au premier plan le pouvoir de l'imagination, en tant que faculté (re)présentative. Car si l'expérience esthétique n'ouvre pas un intérêt théorique, c'est aussi que le rapport à l'objet est sans concept.

Nous ne rapportons pas au moyen de l'entendement la représentation à l'objet en vue d'une connaissance, mais nous la rapportons par l'imagination au sujet et à son sentiment de plaisir et de peine ${ }^{1}$.

Le jugement de goût n'est pas un jugement de connaissance, il n'est pas logique mais esthétique ${ }^{2}$.

Quand on ne dispose pas de concept sous quoi subsumer une représentation donnée, le divers de l'intuition se voit livré à une certaine incertitude, voire à une errance réflexive (comme avec cette figure de Kandinsky (figure 1)).

pour justifier le goût et le jugement (ou critiquer les productions des beaux-arts qui n'y seraient pas conformes), mais les critiques oublient qu'aucun argument, ni bavardage ne suffira à convaincre celui qui ne fait pas par soi seul l'expérience de son propre état de plaisir (soit du libre jeu des facultés), quoiqu'il en soit de toutes règles et de tous préceptes.

${ }^{1}$ E. Kant, Critique de la faculté de juger, op. cit., § 1, p. 49.

${ }^{2}$ Ibid. 


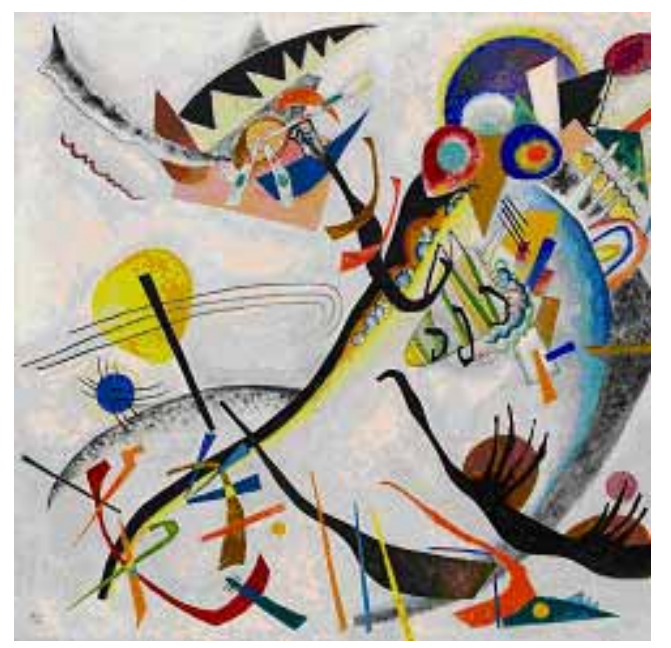

Fig. 1 - Wassily Kandinsky, Segment bleu, $1921(120$ x 140 cm), Musée Guggenheim, New York.

L'entendement n'offrant pas de règle pour composer ce divers, l'imagination est invitée à jouer de sa liberté et elle peut «schématiser sans concept ${ }^{1}$. Ainsi s'annoncent les célèbres analyses sur le libre jeu de l'imagination et de l'entendement, et le plaisir de sentir un accord libre et pourtant réglé des facultés. Rappelons enfin que si le rapport esthétique à l'objet est désintéressé, c'est que l'expérience porte moins sur la chose que sur sa représentation et ne fait aucun cas de son existence réelle ${ }^{2}$. Ou pour le dire avec Husserl, il faut distinguer "l'intérêt pris à la chose (Sache)» et "l'intérêt pris à l'apparition $»^{3}$. En effet, la même chose, qu'elle soit œuvre d'art ou chose naturelle, peut être saisie tantôt dans l'attitude naturelle, tantôt dans une attitude esthétique qui met son existence entre parenthèses et s'abstient de toute prise de position sur la réalité empirique de la chose. Seule compte la présence du visible, la présence de l'apparaître et non celle de la substance réelle $^{4}$. Par ce désintéressement, il s'établit un rapport de distanciation à l'objet autant qu'un rapport de déréalisation qui fait qu'il est en quelque sorte

\footnotetext{
${ }^{1}$ Ibid., § 35, p.122.

${ }^{2}$ Ibid., $\S 2$.

${ }^{3}$ E. Husserl, Hua, XXIII, [145], texte de 1906, trad. fr : Phantasia, Conscience d'image, Souvenir, Grenoble, Jérôme Millon, 2002, p. 168.

${ }^{4}$ Voir aussi par exemple La Lettre de Husserl à Hofmannsthal, du 12 juillet 1907, dans La Part de l'Eil, 1991, Bruxelles, trad. É. Escoubas, p. 13-15.
} 
« hors-jeu » - telle une image. Ce qui fait que l'attitude esthétique installe le spectateur dans une pure vision qui découvre les choses comme phénomènes proposant leur sens immanent, et l'invite à faire l'expérience de l'irréel au sein même de la perception.

On comprend bien cette exigence si on pense à l'exercice singulier de perception qu'appelle le tableau. Car ce n'est pas la chose matérielle accrochée au mur, faite d'une toile et d'un cadre de bois, que nous regardons ${ }^{1}$, mais bien ce qui s'y donne à voir. Et, à son tour, l'image peinte ou gravée nous fascine pour ce qu'elle représente et qui n'est pas là. Soit qu'elle représente quelque chose qui n'est pas actuellement présent, comme un portrait de Marilyn (figure 2), soit quelque chose qui n'existe plus, comme l'image-copie du phare d'Alexandrie (figure 3), ou encore quelque chose qui n'existe pas du tout et s'offre comme une fiction, tel ce paysage idyllique du Titien (figure 4). Ici l'image peinte (qui est aussi une chose réelle, étendue, faite de pigments et de matière colorée) invite à percevoir, au-delà d'ellemême, un objet irréel qui se tient pourtant au sein même de la réalité. C'est ce que Husserl nomme une imagination perceptive et, son corollaire, un fictum perceptif.
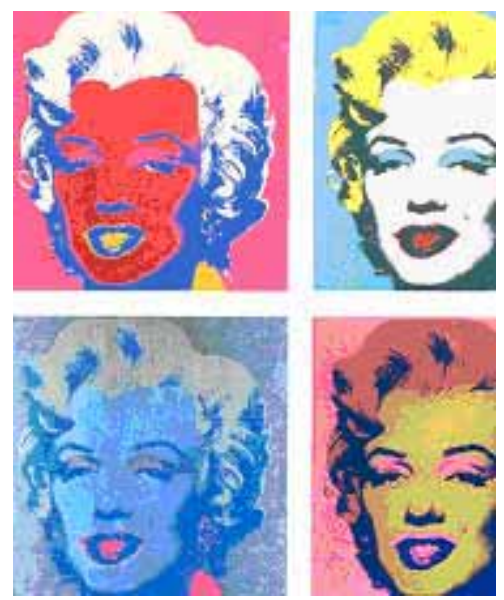

Fig. 2 - Andy Warhol, Marylin Monroe, 1967 (91,5 x 91,5 cm chacune), collection particulière.

\footnotetext{
${ }^{1}$ Cf. R. Ingarden, «Le tableau, l'image picturale », dans Esthétique et ontologie de l'œuvre d'art, Choix de textes 1937-1969, Paris, Vrin, 2011 ; ou encore J. Margolis, «La spécificité ontologique des œuvres d'art» dans D. Lories (dir.), Philosophie analytique et esthétique, Paris, Klincksieck, 2004.
} 


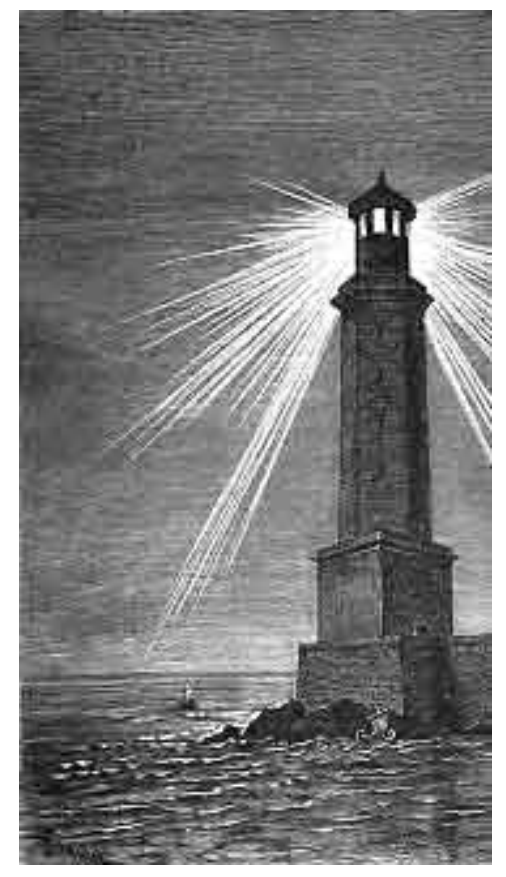

Fig. 3 - Le Phare d'Alexandrie, gravure sur bois de S. Barclay, dans Voyage aux Sept merveilles du monde, par Lucien Augé de Lassus, 1878.

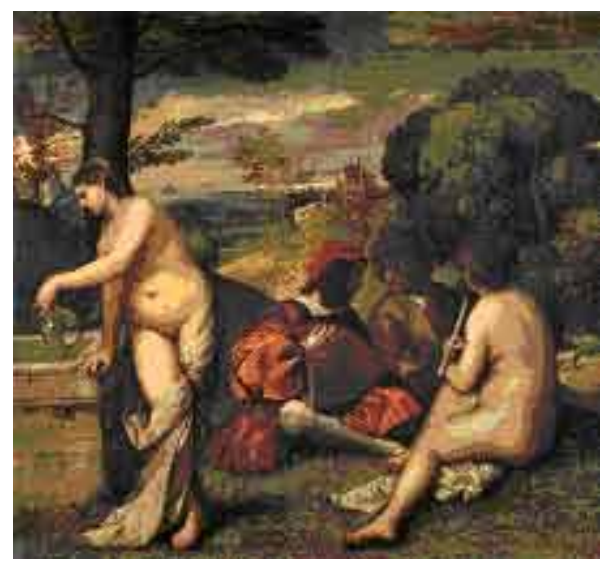

Fig. 4 - Titien, Concert Champêtre, 1509, Musée du Louvre. 
Remarquons que ce n'est pas la vertu du seul tableau, car d'autres formes artistiques nous permettent également d'accéder à un ordre de fiction ou de fantaisie par le biais même de la perception. C'est le cas du théâtre, de la danse, comme aussi bien du jardin régulier qui dessine une broderie végétale.

Dans tous les cas, la réalité de la chose (tableau ou scène) cède le pas à l'irréalité de l'apparaissant. Ici, le perçu est, comme dit Husserl, « en l'air » ${ }^{1}$. «L'image ou l'imaginé ne fait que flotter de manière perceptive » ${ }^{2}$, c'est un quasi-objet donné dans une quasi-expérience, une réalité « comme si ». Pour autant, ce suspens n'est pas une vacuité ni une illusion; le phare, la madone comme la scène pastorale sont bien réellement perçus, "nous faisons bien une expérience, mais nous ne sommes pas dans l'attitude de l'expérience car nous ne prenons pas part à la position d'expérience, les objets sont de simples objets d'imagination, quoique perceptifs $\nu^{3}$. Il ne s'opère aucune prise de position, nul acte thétique posant ou invalidant l'existence de ces objets, leur existence est résolument entre parenthèses, tandis que la perception en est pourtant effective, et le plaisir du "perçu en tant que perçu » appelle sa continuation.

L'attitude esthétique nous plonge ainsi directement dans une expérience perceptive paradoxale où la faculté imaginative s'entremêle à la perception dans un jeu complexe. D'autant que le plaisir esthétique, qui justement est indifférent à l'existence de la chose contemplée, est à son tour d'une nature singulière. Il ne ressemble pas aux autres plaisirs, en particulier aux plaisirs sensuels. Il est pour Kant, « le plaisir de la simple réflexion » c'est-àdire le fait de sentir l'accord ou l'harmonisation spontanée des facultés à l'occasion de la représentation d'un objet.

En conséquence, le rôle de l'imagination s'affirme de manière décisive sur plusieurs plans :

- en tant qu'attitude intentionnelle, en ce qu'elle ouvre un espace de neutralité qui opère par la modification du «comme si » affectée aux différents objets perçus ;

- en tant qu'activité imageante, reproductive ou créative qui produit des images-copies aussi bien que des ficta perceptifs ;

- enfin, elle est décisive en tant que faculté, c'est-à-dire en tant que principe de mouvement ou puissance d'animation qui se traduit dans le jeu touffu du mouvement réfléchissant du côté du spectateur, tout comme dans la

\footnotetext{
${ }^{1}$ Hua, XXIII, p. 485 [514], texte $n^{\circ} 18$, Sur la théorie des intuitions et de leurs modes, vers 1918.

${ }^{2}$ Ibid., p. 479 [506].

${ }^{3}$ Ibid.
} 
création des Idées esthétiques du côté de l'artiste, et dont le sentir est immédiatement un plaisir, et « un plaisir actuel ${ }^{1}$ pour Husserl. De son côté Kant définit cette animation ainsi :

L'âme, en un sens esthétique, désigne le principe vivifiant en l'esprit. Ce par quoi ce principe anime l'esprit - la matière qu'il applique à cet effet — est ce qui donne d'une manière finale un élan aux facultés de l'esprit, c'est-à-dire les incite à un jeu qui se maintient de lui-même et qui même augmente les forces qui y conviennent ${ }^{2}$.

Ce paragraphe relève de manière singulièrement forte la mobilité et l'animation de la vie de l'esprit dans son processus esthétique : il y a quelque chose en l'esprit qui le meut, l'anime et le vivifie en lui proposant une «certaine matière ", certaines représentations ou certaines Idées qui stimulent son activité, la relancent et en quelque sorte l'alimentent par ce va-et-vient entre le positionnel et le suspendu qui entrelace différents types de contenus intuitifs relevant de différentes modalités intentionnelles (image, souvenir, sensation, intuition partielle, visées à vide, acte amorcé et abandonné, kinesthèse, lumière, ambiance, etc.). Cette animation vivifiante tient largement aussi à ce que l'imagination peut, à loisir, faire et défaire, fondre et refondre le divers, les contenus intuitifs dont elle s'empare ou qu'elle produit. Elle peut, à loisir, modifier, varier, transformer d'une façon qui varie à l'infini, car le singulier perçu dans la représentation, dans l'image ou dans le tableau, ne vaut pas tant comme un existant actuel que comme un individu possible. Et plus largement, tous les « imaginés », les objets-images, offrent la dimension du possible, quand bien même ils peuvent se donner comme concrets et individués, c'est encore sous la modification du «comme si ». Par suite, l'expérience esthétique n'a pas besoin de l'existence effective de son objet ni de la règle prégnante d'un concept pour le subsumer.

Si le jeu vivifiant des Idées esthétiques, que ce soit du côté du créateur ou du côté du spectateur, s'exerce de manière privilégiée à partir des œuvres d'art, il peut aussi trouver son occasion sur des réalités naturelles, et Kant le suggère clairement à travers les analyses sur le beau naturel et le sublime dans la nature. Que se passe-t-il lorsque l'expérience esthétique se déroule directement dans l'ordre des choses ou des paysages naturels ? Les caractères de cette expérience que nous venons de rappeler sont-ils toujours opérants ? Plus exactement, les conditions de désintéressement, de suspension de l'existence sont-elles encore recevables?

${ }^{1}$ E. Husserl, ibid., p. 379 (393), vers 1912.

${ }^{2}$ E. Kant, Critique de la faculté de juger, op. cit., § 49. 


\section{Une expérience esthétique de la nature est-elle possible?}

La perception d'un lieu, d'un site ou d'un paysage dans sa réalité concrète et singulière est une expérience qui est d'emblée positionnelle et qui appelle ou impose son appréciation. Comment l'existence d'un paysage pourrait-elle être mise en parenthèses ici ? Puisque c'est précisément à cette existence singulière que nous sommes attachés, c'est elle que nous sommes venus contempler pour faire en quelque sorte la preuve ou le constat de sa réalité même. Le voyageur anglais, le touriste qui passe les Alpes ou l'artiste qui vient en résidence à Rome veulent voir de visu la réalité de la cascade de Tivoli (figure 5) pour s'assurer qu'elle existe bien telle qu'on la dit et telle qu'on la peint.

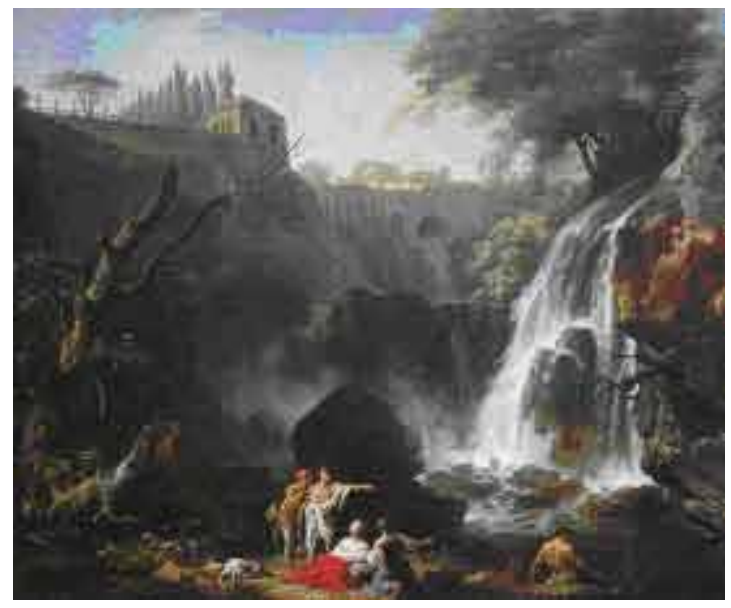

Fig. 5 - Joseph Vernet, Les Cascades de Tivoli et la villa de Mécène, 1740, La Haye, Musée Mauritshuis.

Qui plus est, l'appréhension d'un paysage naturel est une expérience totale où tous les sens sont conviés. Le paysage appelle sa perception, mais aussi son parcours. Et entrer dans l'espace du paysage, c'est rencontrer un espace réel, englobant et non plus figuré et cadré ; c'est du même coup solliciter toutes les ressources du corps, s'ouvrir à l'accueil du vent et de l'air, aux senteurs et aux textures du monde qui troublent et ravissent à la fois, autrement dit, éprouver des émotions, des sensations qui relèvent d'un plaisir ou d'un déplaisir sensuel qui engage la matière même de la chose et non pas son pur apparaître. On peut donc soupçonner que, dans l'expérience de la nature, la réduction qui sous-tend l'attitude esthétique ne soit pas complète, 
que la mise entre parenthèses de l'existence naturelle ne soit pas strictement opérée, qu'elle soit peut-être même impossible dès lors que le paysage invite à son exploration. Il me semble en conséquence que la seule alternative est la suivante :

- soit il n'y a pas d'expérience de la nature qui soit réellement esthétique faute de pouvoir désamorcer les intérêts pris à l'existence des choses ou du paysage,

- soit l'expérience de la nature peut donner lieu à une véritable expérience esthétique, mais cela veut dire que le suspens de l'existence n'est pas la condition fondamentale de l'attitude esthétique, ou qu'il faut la repenser.

Il faut donc revenir sur ce qui fait le propre de cette expérience de la nature et déterminer le rôle que l'imagination y joue.

\section{Que voit-on quand on voit un paysage?}

Husserl répond en 1918 : «Nous laissons l'apparaissant nous plaire comme s'il s'agissait de la réalité ${ }^{1}$. Il considère d'emblée que le paysage agit comme une image ${ }^{2}$, c'est-à-dire invite à être appréhendé selon une attitude esthétique, pour autant qu'on l'aborde de loin, à distance, dans un point de vue cadré ou dans une perspective de surplomb (un prospect, comme disait Montaigne).

Un village lointain. Les maisons «petites maisons ». Ces petites maisons ont a) une taille changée par rapport aux maisons telles que nous les voyons couramment, b) une moindre stéréoscopie, des colorations changées. Elles sont appréhendées comme image ${ }^{3}$.

Autrement dit, on peut voir le paysage comme un tableau, à la manière d'un tableau, comme un fictum perceptif. De fait, le touriste, qui vient voir la cascade de Tivoli, vient chercher un type de vision qui a déjà été largement fixé dans la peinture et dont il s'agit de confirmer la "réalité » tout en l'appréhendant paradoxalement comme si elle était une image. Par extension, Husserl considère que n'importe quel paysage peut donner lieu à un tel jeu perceptif :

\footnotetext{
${ }^{1}$ Hua, XXIII, p. 485 [513-514], texte de 1918.

${ }^{2}$ Hua, XXIII, p. 167 [144], texte de 1906, où Husserl répond à sa question dans la question même : « Pourquoi le paysage agit-il comme une image? ».

${ }^{3}$ Ibid., p. 167 [144]
} 
D'une certaine manière, je peux voir toute chose comme une image. J'inhibe toute croyance effective, je suis sans intérêt envers la réalité effective et je prends la chose en tant qu'image, comme «montagne », c'est-à-dire dans le type d'apparition, comme ayant valeur pour moi ${ }^{1}$.

Ce qui est intéressant, c'est la possibilité évidente pour Husserl de convertir toute perception effective en une phantasia, reproductive ou fictionnelle, ou inversement d'asseoir une imagination perceptive sur l'arrière-fond de perceptions réelles.

Cela signifie que le paysage ou n'importe quelle chose naturelle peut faire l'objet d'une attention esthétique car il est toujours possible de suspendre la thèse naturelle de la foi perceptive ou de la désamorcer en faisant jouer l'imagination au cœur même de la perception, comme pour la tarauder de l'intérieur et convertir la réalité en pur apparaître. Néanmoins, la perception réelle et effective - la perception positionnelle — constitue bien le point de départ du processus esthétique sans que cela gêne en rien l'animation esthétique.

Cela signifie alors aussi que le jeu perceptif est plus complexe que ce que l'on pourrait spontanément supposer, et en tout cas n'est pas binaire, au sens où il y aurait, d'un côté, une perception qui serait exclusivement thétique et, de l'autre, une imagination qui serait purement intérieure, agissant dans la reproduction, la fiction ou la modification de neutralité (le «se transporter en pensée dans »), c'est-à-dire opérant seulement sur des images, des êtres de pure pensée ou de pures apparences. En réalité, dit Husserl, le régime commun de l'expérience est celui de vécus mixtes où les positions d'existence renferment des imaginations, tout comme les imaginations renferment des positions, et le plus souvent, «les imaginations qui viennent habituellement à l'esprit ne sont pas des imaginations pures mais des imaginations impliquées [Hineinphantasien — qui s'insèrent en dedans] qui imaginent un fictum dans un fragment de réalité d'expérience intuitive, ou se projettent en imagination dans une réalité obscurément posée $»^{2}$. Inversement, il n'est pas vrai que la perception soit déliée de toute imagination puisque la simple anticipation suppose ce prolongement imaginatif du perçu en direction de ses horizons internes et externes.

\footnotetext{
${ }^{1}$ Hua, XXIII, p. 552 [593], Appendice LXV, vers 1920. Et inversement : « Il en va de même si un intérêt de contemplation pure nous conduit à la réalité effective et au caractéristique et typique d'une tranche de monde donnée. Une ferme caractéristique de la Forêt Noire, un paysage de la Forêt Noire, etc. », p. 510 [541].

${ }^{2}$ Hua XXIII, p. 482 [509], texte $n^{\circ} 18$, Sur la théorie des intuitions et de leurs modes, vers 1918.
} 
Autrement dit, les échanges se font dans les deux sens et il est toujours possible d'amorcer une attitude réduite ou modifiée à partir d'une position d'existence. Mieux, c'est aussi ce qui favorise un jeu d'aller-retour de l'image à la chose, de l'apparaissant à la réalité perçue, selon un cercle assez ludique : car si «l'apparaissant nous plaît comme s'il était réalité », il faut entendre ici — dans le cas du paysage — qu'il est bien d'abord perçu et appréhendé comme réalité actuelle et, qu'ensuite, la distance ou le cadrage permettent de désamorcer sa réalité pour poser le perçu en tant qu'image. On passe ainsi d'une conscience positionnelle à une conscience de «comme si », et parallèlement d'une conscience de "comme si » à une réalité qui peut encore jouer à être une image-copie d'un tableau, etc. Il s'ensuit un jeu de fascination qui oscille entre position et suspens, et ce d'autant plus qu'il y a certaines choses, selon Husserl, qui «s'avèrent propres à provoquer une double aperception, c'est-à-dire où la perception vire facilement en d'autres types d'apparitions perceptives $\gg{ }^{1}$. Et c'est bien le cas du paysage qui offre souvent la possibilité du panorama, du cadrage et du recul, et qui bénéficie aussi de l'héritage de la culture artistique de la peinture de paysage comme de la photographie, qui nous a appris à voir, à artialiser pour reprendre le mot d'Alain Roger ${ }^{2}$. Ces habitudes artistiques ou esthétisantes font que, devant un paysage, on est rapidement enclin à associer des images à la réalité, des images aux images, des images aux souvenirs, etc. La perception accueille l'imagination tout comme le jeu imaginatif s'empare du perçu et ce faisant en désamorce l'effectivité.

Il faut insister ici sur le fait que la conscience imaginative et esthétique opère sur le sol de l'expérience et des concordances d'horizon que suppose l'expérience de la nature, et que même si cette dimension passe à l'arrièreplan, elle n'est pas éliminable. C'est même elle qui donne lieu à ce jeu d'ambivalence entre les attitudes, positionnelle et suspendue à la fois. Autrement dit, la réduction n'est pas complète mais la conscience esthétique est néanmoins amorcée. Il faut en tirer la conséquence avec Husserl que la mise hors circuit de toute prise de position (qui est aussi le principe de la méthode phénoménologique) "n'est pas en soi un acte esthétique ${ }^{3}$ et, corrélativement, que tout paysage peut également susciter un plaisir esthétique, quel que soit son mode d'être et son mode positionnel : qu'il soit réel ou fictif, qu'il soit mis entre parenthèses ou maintenu dans sa thèse.

\footnotetext{
${ }^{1}$ Ibid., p. 488 [518].

${ }^{2}$ A. Roger, Court traité du paysage, Paris, Gallimard, 1997.

${ }^{3}$ Hua XXIII, p. 377 [391], texte $n^{\circ} 15$, Modes de reproduction et phantasia. Conscience d'image (également en relation avec les prises d'attitude) (mars-avril 1912).
} 


\section{4. À quelle condition une conscience esthétique est-elle possible ?}

Quel est le véritable ressort de la conscience esthétique, si ce n'est pas la suspension des positions? Et « qu'en est-il à présent de la conscience esthétique quant à son insensibilité envers l'être et le non être ? ${ }^{1}$. Est-ce bien la condition de l'attitude esthétique?

Il n'est pas correct de dire que la conscience esthétique est dirigée sur l'apparaissant, et par conséquent sur ce qui est à décrire, hors considération de l'être et du non-être, mais elle y est dirigée sur le type d'apparition en cause. Lui seul est esthétique ${ }^{2}$.

Husserl introduit par là une dimension qui n'a pas encore été soulignée alors qu'elle est sans doute la clef de ces changements d'attitude intentionnelle. Si l'attitude esthétique trouve un appui privilégié dans la conscience d'image, c'est d'abord parce qu'elle s'attache à un certain mode d'apparition ou type d'apparition, non pas seulement au fait de ne pas prendre position vis-à-vis de la réalité. Que faut-il entendre par mode d'apparition (Erscheinungsweise) ? Husserl donne quelques indications dès le texte de 1906 :

Différentes apparitions du même objet ne sont pas d'égale valeur; mise en place des vases, des cendriers, etc. dans le salon. Quelle est la plus belle position? C'est donc bien esthétique. Là l'apparition la plus favorable est choisie ${ }^{3}$.

Malgré le prosaïsme de l'exemple on comprend très bien que la disposition, l'orientation, l'éclairage, les angles de vue, la mise en scène des objets dans une pièce ne composent pas le même effet, précisément, le même type d'apparition. Le type d'apparition, c'est « le comment de l'apparaissant », ou encore l'ensemble des dispositifs (le profil, l'aspect, le style, la lumière, etc.) à travers lesquels l'objet apparaissant se figure, soit tout ce qui participe à un mode de donnée qui peut provoquer des sentiments, des réactions affectives, du préférable, par suite du plaisir et de la valeur. Par où l'on retrouve aussi un aspect que nous étions en train d'oublier et que Kant avait pourtant bien mis au cœur du processus esthétique, à savoir le plaisir pris à l'apparition. Ce qui rappelle encore que l'expérience esthétique ne va pas sans une intention

\footnotetext{
${ }^{1}$ Ibid.

${ }^{2}$ Ibid., p. 377 [391].

${ }^{3}$ Op. cit., p. 168 [145].
} 
évaluative (et non pas doxique ou théorique ${ }^{1}$ ) qui prend appui sur le type d'apparition sous lequel une chose se figure. Ainsi, ce qui est véritablement fondateur de la conscience esthétique, c'est l'impact du mode d'apparition, et non pas le fait de mettre la réalité en suspens.

Et Husserl note d'ailleurs que «lorsque je considère la nature sous l'angle esthétique, celle-ci demeure pour moi cette réalité effective déterminée $»^{2}$. Nul besoin alors d'en faire une image, parce que les prises de position ne portent pas tant sur l'objet lui-même (le paysage naturel) que sur les sentiments induits par les modes d'apparition et la réflexion sur le rapport entre ces modes et l'apparaissant :

Je ne vis pas dans la conscience de cette réalité effective...mais je vis dans des sentiments qui sont déterminés par le type d'apparition, et de surcroît par tel ou tel type de conscience de la nature, et qui, au regard de ces types de donnée « subjectifs » et dans le passage de l'attitude d'objet à l'attitude réflexive et inversement, sont conscients comme déterminités affectives de l'objectal lui-même'.

Autrement dit, ce qui est effectivement vécu en prise d'attitude, c'est un sentiment, et un sentiment qui ne porte sur l'objet que "par égard pour l'apparition ${ }^{4}$, donc qui ne porte pas sur l'objet lui-même mais en quelque sorte le traverse pour atteindre l'apparition ou les séries d'apparitions possibles, jusqu'à saisir celle qui plaît ou qui réjouit; et, au fond, peu importe que l'objet existe réellement ou en image. Ce qui intéresse esthétiquement, ajoute encore Husserl dans les années vingt, c'est «l'objectité dans le comment $»^{5}$, c'est-à-dire dans le « comment de sa figuration », et la possibilité d'en jouer, de faire varier cet apparaître à travers les types d'apparition.

Ici se tient le ressort fondamental de l'expérience esthétique, et c'est par là que Husserl, comme Kant avant lui, distingue la conscience esthétique de la conscience naturaliste, et plus largement de l'attitude théorique et positionnelle en général qui cherche prioritairement à identifier l'objet en tant que le même, quels que soient ses modes d'apparition :

${ }^{1}$ Hua XXIII, p. 373 [386], texte $\mathrm{n}^{\circ} 15$ : Modes de reproduction et phantasia, conscience d'image (également en relation avec les prises d'attitude), mars-avril 1912.

${ }^{2}$ Ibid., p. 377 [391].

${ }^{3}$ Ibid., p. 377 [391].

${ }^{4}$ Ibid., p. 378 [392].

${ }^{5}$ Appendice LXIV, p. 551 [591]. 
Que l'objet apparaisse dans telle ou telle orientation, c'est tout un pour la prise d'attitude réaliste (...) Mais esthétiquement ce n'est pas tout un. L'évaluation esthétique dépend par essence de la distinction entre conscience d'un objet en général et type d'apparition de l'objet ${ }^{1}$.

Comme on voit, ce n'est pas tant la suspension de l'existence qui est décisive que le rapport à l'objet, et à ce qui motive le regard sur l'objet. Ou bien l'objet est visé et saisi en vue de son identification théorique comme étant le même, quels que soient ses modes d'apparition, de manière à servir des intérêts cognitifs ou pratiques. Ou bien l'objet est appréhendé exclusivement par rapport à ses modes d'apparition, plus ou moins heureux ou réjouissants. Ce que Husserl résume simplement en disant : «La considération esthétique exige l'exclusion de l'intérêt théorique $»^{2}$, ou pour le dire en termes kantiens, le rapport à l'apparaissant est libéré de son rapport à un concept :

L'intérêt ne concerne pas l'objet en tant que membre du monde effectivement réel, selon ses propriétés objectales, ses relations, etc., mais précisément l'apparition seulement ${ }^{3}$.

Comme Kant, Husserl marque très clairement une scission entre attitude esthétique et attitude cognitive au sens large, seul le «comment de l'apparaître » motive la conscience esthétique. Ce qui signifie aussi qu'un site ou un paysage naturel peut donner lieu à une appréhension esthétique, sans solliciter aucune connaissance, sans favoriser une volonté d'acquisition immobilière, sans offrir de qualités agricoles ou botaniques, bref sans stimuler aucun intérêt théorique ou pratique. Le beau paysage ne l'est pas par ses qualités géomorphologiques mais par l'angle de vision, l'orientation, la lumière, la mise en perspective, etc., autant d'éléments qui sont indifférents à la réalité physique, chimique, géologique ou tectonique de l'apparaissant. Par là, nous devons faire place à une objection actuelle venue d'une discipline nouvelle, l'esthétique environnementale.

\section{Approche esthétique et approche cognitive du paysage}

Il s'agit d'une conception anglo-américaine - je me réfère ici principalement aux travaux d'Allen Carlson ${ }^{1}$ — une conception de l'esthétique qui

\footnotetext{
${ }^{1}$ Hua XXIII, p. 375 [388].

${ }^{2}$ Hua XXIII, p. 550 [591], Appendice LXIV, vers 1921 ou 1924.

${ }^{3}$ Hua, XXIII, p. 168 [144], texte de 1906.
} 
entend se démarquer de la tradition philosophique, qui ne prétend ni à une philosophie de l'art ni à une théorie du beau ni à une théorie du goût, mais cherche à étendre l'appréciation esthétique aux environnements naturels et urbains. Il y va d'une approche naturaliste qui pose en principe que l'appréciation des objets et des environnements naturels exige, à titre de condition nécessaire, la connaissance que nous en procurent les sciences de la nature. L'appréciation esthétique suppose de connaître la nature de l'objet, ainsi que l'histoire de sa production. Le rapport au paysage naturel n'appelle pas une expérience sensible et imaginative de l'objet, mais une connaissance objective de ce qu'il est et de la façon dont il a été produit. Autrement dit, pour apprécier un paysage naturel, tel qu'une crique de schiste et de galets sur le littoral nord de l'île d'Ouessant (figure 6), il faudrait connaître la nature des roches et l'histoire géologique de leur formation expliquant leurs strates, leurs formes et leurs dispositions. Seules ces connaissances nous permettent d'apprécier les qualités et les propriétés de ce paysage, d'en apprécier « l'ordre ou la raison naturelle » qui seuls décident de la valeur positive de l'organisation naturelle. La thèse est claire : faute de connaissance géographique et géologique, on ne peut pas avoir une perception esthétique d'un paysage, ni l'apprécier à sa juste valeur. C'est évidemment problématique. Qu'en est-il de l'appréciation de la nature avant l'avènement des sciences naturelles et physiques modernes?

Pour autant, tout le monde convient que le savoir permet de mieux voir ; qu'une certaine connaissance permet de mieux « comprendre » un paysage, de mieux l'analyser, tout comme par analogie avec l'objet artistique, la connaissance d'un artiste, de son époque, de son style permet de mieux percevoir et de mieux juger ses œuvres. Mais cette condition, pour importante qu'elle soit, n'est ni nécessaire ni suffisante.

D'une part, cette conception confond ce qui « enrichit» la perception, l'instruit ou l'affine, et ce qui provoque l'attitude esthétique elle-même. D'autre part, la volonté de saisir les propriétés, les qualités, les raisons naturelles d'un paysage reconduit l'appréhension de ce paysage à ces éléments constituants et aux divers concepts qui peuvent en rendre compte, autrement dit, reconduit à la démarche d'un jugement déterminant. Or, ce que Kant, comme Hume déjà, puis Husserl ont largement et solidement mis en évidence, c'est le fait que la perception esthétique sollicite la démarche inhabituelle du jugement réfléchissant : nous ne jugeons pas l'objet d'après ses qualités réelles ou apparentes, mais nous rapportons cette représentation

${ }^{1}$ Cf. A. Carlson, Nature \& Landscape. An Introduction to Environmental Aesthetics, New York, Columbia University Press, 2009. 
au sentiment de plaisir ou de peine sans la médiation d'aucun concept ${ }^{1}$. Kant en donne un exemple très significatif :

Des fleurs sont de libres beautés naturelles, ce que doit être une fleur peu le savent hormis le botaniste et même celui-ci, qui reconnaît dans la fleur l'organe de la fécondation de la plante, ne prend pas garde à cette fin naturelle quand il en juge suivant le goût ${ }^{2}$.

On mesure tout à fait la concordance avec l'exemple du météorologiste de Stolnitz, rappelé plus haut, et on trouve autant de formulations comparables chez Husserl :

Je ne m'intéresse pas à l'objet effectivement réel - l'intention ne porte pas sur son remplissement de tous côtés en tant qu'intention de connaissance, sur ce qu'il est trait pour trait et absolument. Il ne s'agit donc pas de la nature en général où cette chose me fait entrer d'après son être ${ }^{3}$.

Prenons alors un exemple (figure 7) pour cerner la différence entre ces attitudes.

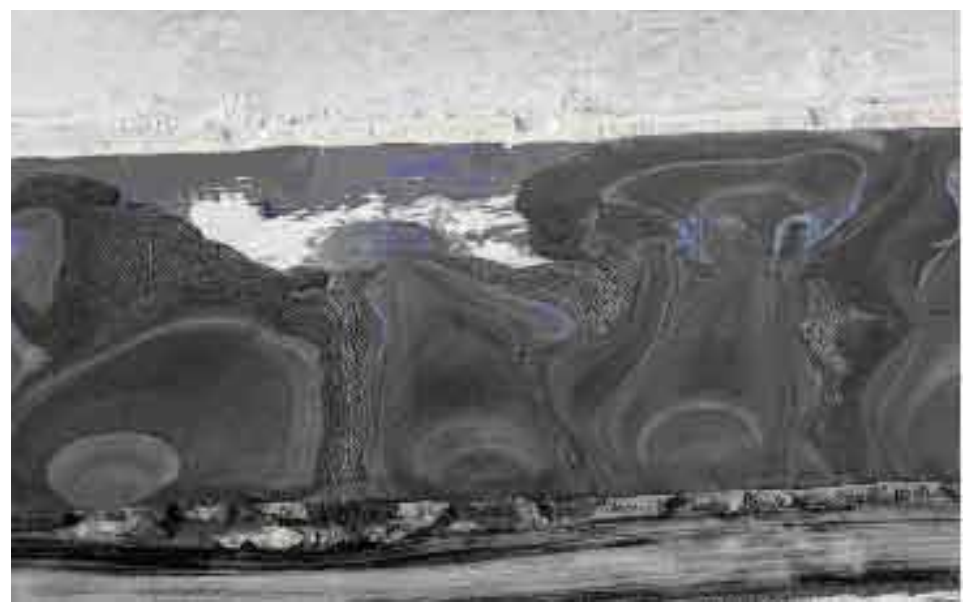

Fig. 7 - (C) Alex McLean, Photographie aérienne, Lac Mildred, Canada, 2014.

\footnotetext{
${ }^{1}$ « Le jugement s'appelle esthétique parce que son principe déterminant n'est pas un concept, mais le sentiment (du sens interne) de l'accord dans le jeu des facultés de l'esprit dans la mesure où celui-ci ne peut qu'être senti » (E. Kant, Critique de la faculté de juger, op. cit., § 15, p. 70).

${ }^{2}$ Ibid., p. 71.

${ }^{3}$ Hua XXIII, p. 507 [538].
} 


\section{Un paysage à interpréter}

Que voit-on exactement sur cette photographie aérienne prise par Alex MacLean ${ }^{1}$ ? Que voit-on et comment voit-on ?

Le jeu des lignes et des couleurs est assez vertigineux, et la perspective à vol d'oiseau perturbe les habitudes visuelles : on perçoit une ampleur, une immensité, une hauteur, mais de quoi ? On ne sait pas exactement ce qu'on voit. Pourtant l'indication du Canada laisse penser à des montagnes, à des escarpements, des plongées de rochers vers la mer, mais cela reste indécis. En ce sens, l'absence de concept s'éprouve assez vite et l'incertitude stimule l'imagination, provoque une recherche, une interprétation des éléments.

Si maintenant on veut éclairer le sens de cette image par le savoir, on peut préciser sa situation géographique et son objet exact: il s'agit du site minier de Syncrude, au bord du lac Mildred et de zones d'écoulement de goudron de pétrole. Autrement dit, par-delà la belle configuration plastique, se tient un vaste gisement de pétrole avec ses excès, sa pollution, sa puanteur. Le savoir exact de la nature et de l'histoire de ce gisement change-t-il résolument notre perception? Savoir que l'on contemple un épanchement de nappes de pollution nous empêche-t-il de trouver cette image belle ? Ou inversement, le fait de le savoir peut-il augmenter notre plaisir?

Il me semble que dans les deux cas, le savoir ne change rien au plaisir du "comment de l'apparition », à sa figure énigmatique, harmonieuse et inquiétante à la fois, et ces informations n'apportent qu'une étrangeté anecdotique qui ne modifie pas l'appréhension esthétique et imaginative qui peut s'opérer ici. Le paysage, pour réel et naturel qu'il soit, peut aussi se saisir comme une pure forme plastique, quoiqu'il en soit des concepts sous lesquels il peut être subsumé.

Mais sans doute pourrait-on émettre une objection : à savoir que cette vue fonctionne trop largement comme un tableau, et qu'en ce sens elle est plus artificielle que naturelle, qu'elle est davantage une œuvre d'art qu'un paysage naturel. L'argument est recevable. Recommençons alors l'expérience sur un paysage non composé, saisi frontalement, directement, tel qu'il s'offre. Revenons alors à notre crique sur l'île de Ouessant. Je propose de décliner différentes lectures et appréhensions que ce paysage (figure 6) peut susciter.

\footnotetext{
${ }^{1}$ Alex MacLean, photographe américain né en 1947, artiste et aviateur, il travaille également en liaison avec des projets d'aménagement urbain.
}

382 


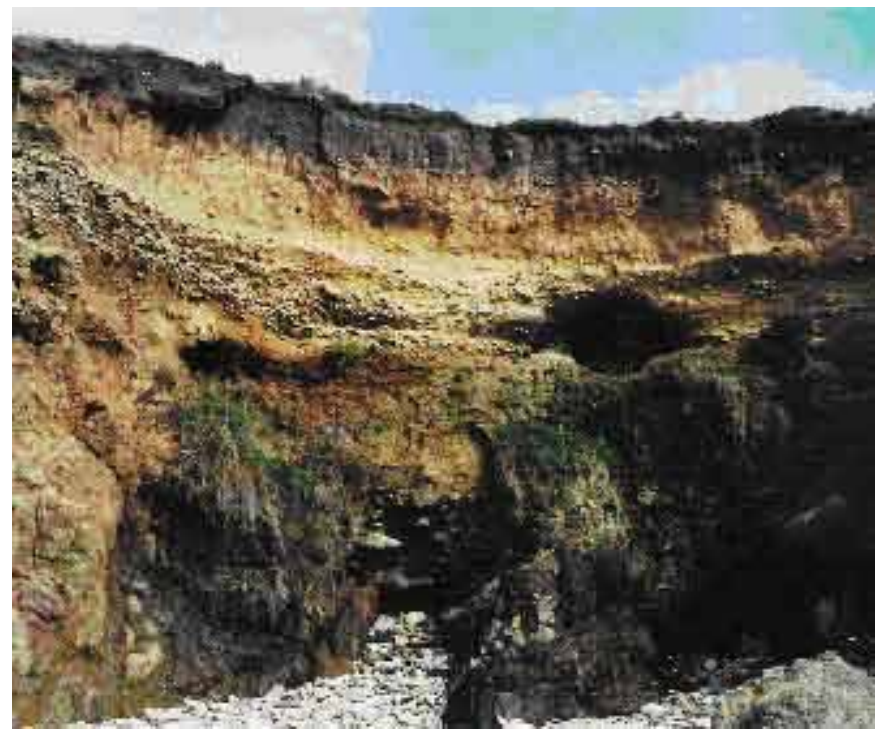

Fig. 6 - C Olivier Planchon, Côte nord de l'île d'Ouessant — Bretagne, France.

\section{Lecture géographique d'un paysage}

Cette crique, comme bien d'autres sur le littoral breton, est favorable à une lecture scientifique précise car elle peut être vue comme une coupe géologique et, en ce cas, voir suppose un savoir réel. Que verrait ici un regard instruit par les sciences naturelles par opposition à un regard "naïvement » esthétique?

Pour un géographe ${ }^{1}$, les différentes strates sédimentaires qui composent la paroi se distinguent immédiatement par leur positionnement, leur couleur et leur structuration. Ces aspects fonctionnent comme des indices permettant de dater, au moins approximativement, la période de formation de ces strates, et d'en présumer les causes. Ainsi, comme le montre la figure suivante (figure 8), la partie inférieure de cette coupe offre des galets qui sont des traces de l'époque Eémienne (autour de cent trente mille ans avant notre

\footnotetext{
${ }^{1}$ Je remercie Olivier Planchon (géographe, climatologue, chercheur au CNRS et à Rennes 2) de m'avoir fait part de sa lecture géo-climatique et de ses explications résumées sur l'image suivante reprenant successivement les différentes strates de la coupe.
} 
ère), tandis que la partie supérieure présente un pavage nival qui n'offre pas la même disposition des galets et indique une période de glaciation, etc.

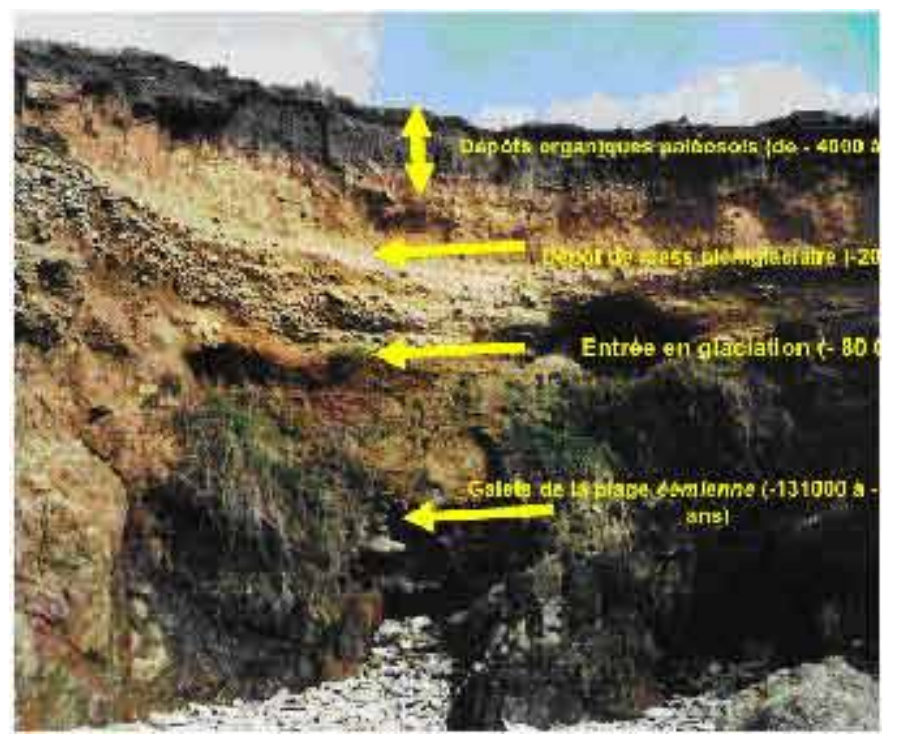

Fig. 8 - Ouessant, une coupe géomorphologique remarquable. Photo et texte (C) Olivier Planchon.

Le géographe est donc bien en mesure, comme le requiert Carlson, de percevoir l'histoire et la genèse de ce paysage. S'agit-il pour autant d'une appréciation esthétique ? Rien ne l'exclut bien sûr, mais est-elle strictement appelée par ce savoir lui-même ?

Car la maitrise de ce savoir et son application à cette paroi rocheuse induisent sans nul doute un plaisir certain, mais un plaisir qui est premièrement intellectuel : " le plaisir de connaître et de reconnaître », comme dirait Aristote, de tester sa science, sa compétence et de l'augmenter, et ce plaisir est assurément un plaisir intense. Mais Carlson va plus loin et suppose que la connaissance théorique est en elle-même déjà esthétiquement plaisante. Il me semble qu'il y a là un saut et un déplacement des modes d'appréhension.

\section{Plaisir esthétique et plaisir théorique}

En effet, il y a deux choses à distinguer. D'une part, et c'est indéniable, le fait que le savoir procure du plaisir, le plaisir intellectuel d'augmenter la 
connaissance, qui peut aussi être un plaisir personnel de se réjouir de progresser. D'autre part, le fait que ce savoir offre une dimension esthétiquement plaisante, et c'est autre chose.

Cela peut en effet être le cas, comme lorsqu'un mathématicien juge qu'une démonstration est « belle » ou plus belle qu'une autre car elle est plus simple, plus élégante, plus directe ou plus intuitive. Mais ce faisant, il ne juge pas du point de vue du contenu conceptuel ou argumentatif, mais plutôt du point de vue de la forme et de la manière dont une démonstration se compose - même si bien sûr ce regard suppose de comprendre et de connaître le sens du raisonnement mathématique. Il regarde le "comment de la donation » d'une démonstration et, s'il en éprouve de la joie, c'est «par égard pour la beauté des relations, démonstrations ou théories mathématiques » ${ }^{1}$. Par là même, Husserl distingue clairement les deux attitudes :

Considérer un paysage, une théorie sous l'angle esthétique, examiner des pensées, sans prendre attitude envers elles, rien que pour apprécier leur caractère esthétique - les deux peuvent ici passer l'un dans l'autre : la considération esthétique exige l'exclusion de l'intérêt théorique, l'attitude théorique doit céder à l'attitude esthétique ${ }^{2}$.

On peut toujours distinguer la perception en tant que prise de connaissance (et qui n'est pas esthétique) et la perception en tant qu'elle se fait sensible aux types d'apparition, mais ce qui complique les choses, c'est que, dans le premier cas, la conscience d'objet peut aussi être doublée d'une intuition des types d'apparition, et ceux-ci «peuvent avoir déjà et en même temps suscité des sentiments $\|^{3}$. C'est pourquoi ces plaisirs sont très proches, à la fois dans le temps de leur épreuve, et sans doute aussi dans leur nature. Et Husserl le reconnaît explicitement, en notant : «L'intérêt théorique est parent du plaisir esthétique $»^{4}$.

Sur quoi cette parenté repose-t-elle exactement? Husserl s'en explique d'une formule lapidaire : "Joie à l'intuition du concret " ${ }^{5}$. La racine commune, c'est le plaisir de l'intuition, cette vision qui donne la chose même et où le voir et le comprendre sont concomitants. On peut noter que Husserl est en cela au plus près de la conception développée par Aristote, au chapitre 4 de la Poétique, lorsqu'il articule et entrelace le goût du savoir au goût des

\footnotetext{
${ }^{1}$ Hua XXIII, p. 378 [392].

${ }^{2}$ Hua XXIII, p. 550 [591].

${ }^{3}$ Hua XXIII, p. 378 [392].

${ }^{4}$ Ibid.

${ }^{5}$ Hua XXIII, p. 510 [541], Appendice LIX.
} 
images. L'homme aime naturellement savoir et il aime naturellement voir des images, car en voyant, il reconnaît et conclut : «celui-là c'est lui ». Autrement dit, l'image est riche en informations, en signes ou en indices, elle donne à voir et à savoir en même temps. C'est précisément le sens grec de la théôria que Husserl reprend ici, car étant formée sur la racine du verbe orao, la notion de théôria renvoie étymologiquement à l'œil, à la vision optique avant de désigner la vue intellectuelle. À cet égard, la peinture figurative (et mimétique) et les arts réalistes, tout comme la biographie, le document historique, la planche anatomique ou la coupe géologique, apportent une joie intellectuelle : " Joie à l'intuitionner qui comprend, corrélativement intérêt théorique à regarder au-dedans, à comprendre le type concret appartenant à un temps ». Toutefois, souligne Husserl, «Il n'est pas ici question de beauté... C'est une partie de la connaissance scientifique », car l'art réaliste peut servir «à son tour d'intermédiaire au savoir ${ }^{1}$. Le plaisir trouve sa place de part et d'autre, mais il n'offre pas exactement le même sens, quelle que soit l'étroitesse des liens et des interactions. À sa manière Aristote le signalait déjà en distinguant le plaisir issu de la connaissance (et de la reconnaissance) du plaisir qui vient « du fini de la composition ou des couleurs $»^{2}$ lorsqu'on ne connaît pas l'objet représenté ; soit un plaisir proprement esthétique qui passe par-dessus les concepts et les identifications, et se repait de formes et de lignes, de disposition et de composition.

\section{Lecture esthétique d'un paysage}

En conséquence, on peut admettre un deuxième niveau de lecture ou de perception pour notre paroi rocheuse à Ouessant, qui serait purement plastique, par suite seulement esthétique.

Lignes, strates, graduations, galets, mousses, etc., tout cela compose une harmonie de formes, de matières et de couleurs qui peut se laisser apprécier pour elle-même sans concept et sans position d'existence. La perception, qui commence dans l'attitude positionnelle, peut se focaliser sur les modes d'apparition, s'attacher aux lignes et aux figures comme à de pures formes, à un décor ou un tableau. Depuis la perception :

Je peux revenir à l'attitude esthétique; je peux considérer la réalité effective comme si elle était une image, ou bien au contraire entrer dans l'attitude de la

${ }^{1}$ Ibid.

${ }^{2}$ Aristote, La Poétique, Paris, Seuil, 1980, 48b 18. 
réalité effective comme si, pour à partir de là au lieu d'être dans l'attitude du poser l'être comme si, passer de nouveau dans l'abstention de l'attitude d'être et de la direction du regard sur le type de donnée. Pour l'attitude esthétique, l'essentiel n'est donc pas non plus la phantasia mais l'attitude envers ce qui intéresse esthétiquement, l'objectité dans le comment ${ }^{1}$.

C'est à partir de ce jeu d'aller-retour entre les attitudes et les modes d'apparition que s'installe la conscience esthétique, plus précisément grâce à la réflexion sur les modes d'apparition. Cette précision est capitale parce qu'il ne s'agit pas tant de s'attacher aux seules formes objectales du paysage, comme à un graphisme pur - car ce serait encore en quelque manière une attitude intéressée à un enjeu théorique - que de faire vivre le sentiment esthétique, c'est-à-dire de faire sentir la mobilité de l'imagination et des changements d'attitude. Autrement dit, ce qui est déterminant ici, c'est moins l'imagination en tant que production ou produit — «l'essentiel n'est pas la phantasia » dit Husserl — que l'imagination en tant qu'intentionnalité qui permet de jouer avec les modes d'apparition; soit l'imagination qui réfléchit et se fait réfléchissante, qui fait varier les régimes d'apparaître, repère les inflexions que cela induit sur l'objet, les allers-retours entre objet, apparition et mode de donation, corrélativement les jeux entre les sentiments vécus et les sentiments feints, la joie réelle et la quasi joie :

Je n'accomplis pas les sentiments, je ne vis pas en eux, si je ne réfléchis pas sur le type d'apparition. L'apparition est apparition de l'objet, l'objet est objet dans l'apparition. Je dois, du vivre dans l'apparaître, revenir à l'apparition et inversement, et alors le sentiment devient vivace ${ }^{2}$.

Va-et-vient, réflexion entre les modes de donation et les objets apparaissant, on retrouve ici une description comparable à celle de Kant et, finalement, une conclusion aussi comparable en ce que c'est dans la réflexion seule (le jugement réfléchissant) que l'imagination trouve son régime proprement esthétique. Par suite, cette réflexion est aussi ce qui contient comme « une force de motivation », car le plaisir pris aux aspects et aux modes d'apparition se renforce, devient vivace dans la confrontation et la variation. On retrouve ce même vocabulaire de la vie, de la joie vivifiante qui était déjà chez Kant la marque du plaisir esthétique, par opposition à la jouissance seulement sensorielle qui « ne laisse rien pour l'Idée », rien pour la réflexion,

\footnotetext{
${ }^{1}$ Hua XXIII, p. 551[591], Appendice LXIV, vers 1921 ou 1924.

${ }^{2}$ Hua, XXIII, p. 376 [389], texte $\mathrm{n}^{\circ} 15$ (h), Modes de reproduction et phantasia (1912).
} 
mais s'épuise vite, "émousse l'intelligence» et finit par «rendre l'âme mécontente de soi $»^{1}$. Au contraire, l'animation esthétique se renforce dans son jeu même de variation réfléchissante sur les modes d'apparition. Elle y gagne un plaisir esthétique pur, si l'on veut parler comme Kant, et qui n'emprunte pas aux savoirs des sciences naturelles.

\section{Lecture métaphysique d'un paysage}

On peut encore orienter l'expérience esthétique menée sur ce paysage naturel dans une tout autre direction en laissant l'imagination se confronter à une réflexion plus métaphysique, stimulée par les Idées de la raison. En effet, la variation sur les modes d'apparition redonne aussi sa dimension concrète et matérielle à l'objet - ici à la matière des roches, des galets, de la terre qui, justement déliée de tout intérêt pratique et scientifique, en vient à paraître pour elle-même dans sa nudité tellurique et sa puissance primitive. Le paysage esthétiquement perçu peut dès lors entraîner l'imagination perceptive à s'abimer dans une vision vertigineuse, une vision "dépaysante " comme dit Lyotard, qui nous fait basculer dans la brutalité de l'originaire. En effet, Jean-François Lyotard remarquait dans Scapeland, que «le paysage est le contraire d'un lieu $»^{2}$, il est ce qui survient et nous sidère : il est l'irruption de «la seule présence visible, ici et maintenant». C'est même trop de présence, car dit-il, c'est « une affaire de matière », la mise en présence avec la réalité nue du monde, avec ses formes informes et chaotiques qui créent le vertige et la désorientation.

Le paysage ainsi dévoilé peut avoir la puissance d'un choc ontologique, car dans le jeu de variations des modes d'apparition, la conscience peut aussi se laisser fasciner par une saisie de plus en plus focalisée qui la confronte à une étrangeté radicale, comme à une hylé primordiale qui se tiendrait là avant toute édification du sens. Et, ce jeu réfléchissant mobilise les Idées de la raison et à leur suite des idées esthétiques qui déploient de multiples visions fantasmatiques autour de l'origine, de la création, de la fin des temps ou de la mort. On retrouve fréquemment cette possibilité dans les photographies de paysage, mais de manière plus privilégiée encore dans les photographies de montagne (figure 9) (de sols, de glaciers, de bassins versants) qui favorisent à nouveau ce dépassement de la perception position-

\footnotetext{
${ }^{1}$ E. Kant, Critique de la faculté de juger, op. cit., § 52, p. 153.

2 J.-F. Lyotard, «Scapeland », Revue des sciences humaines, tome 30, n²09, Janmars 1988, p. 40.
} 
nelle au profit d'un jeu d'appréhension mobile et dynamique entre les modes d'apparition. Certains paysages dont l'indétermination, ou l'excès tout à la fois qualitatif et quantitatif, annule tout quod, égare tout concept, mais motive le jeu des idées et des imaginations, entre réalité et fictum, entre idéalité et possibilité. Ces perceptions en présence peuvent s'ouvrir, dans le jeu esthétique et par-delà toute connaissance naturaliste, à une présentification phantasmée de la matière originaire de notre rapport au monde, faisant vivre des sentiments actuels — trouble, frémissement ou angoisse — qui s'apparentent singulièrement à l'émotion du sublime.

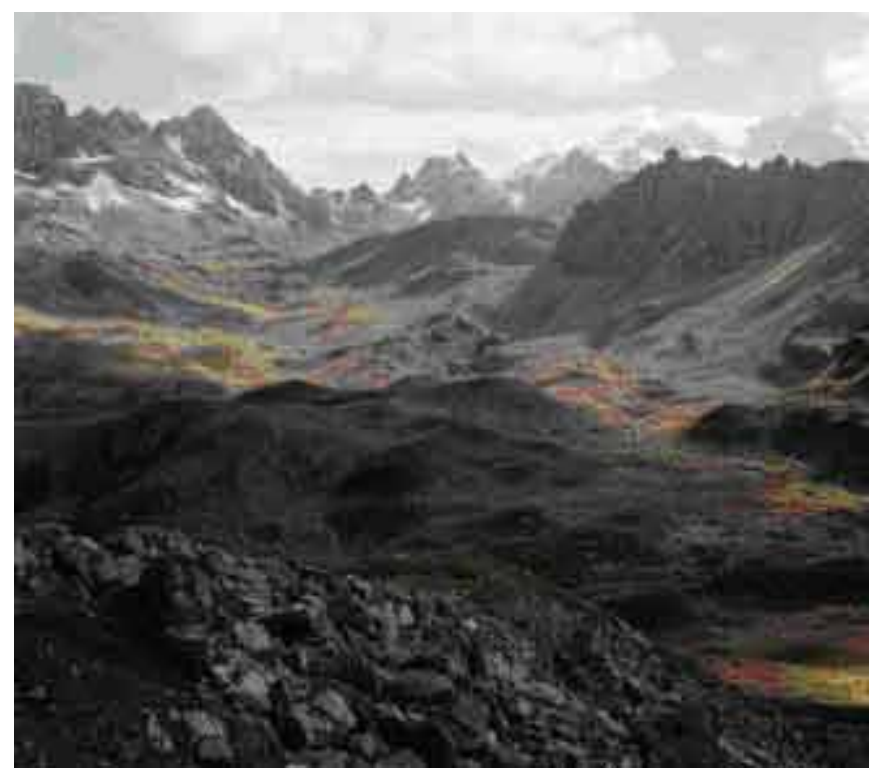

Fig 9 - (c) Beatrix Von Conta, Observatoire photographique du paysage, Saint-Bon Tarentaise, 2500 m, Savoie, 2006

Le paysage, naturel ou artistique, invite à vivre des sentiments esthétiques qui peuvent aussi bien prendre la forme ravie d'une lointaine familiarité, le plaisir vivifiant de ressaisir quelque chose d'une connaturalité de notre appartenance au monde, comme dirait Mikel Dufrenne ${ }^{1}$ ou à l'inverse

\footnotetext{
${ }^{1}$ M. Dufrenne, Le Poétique, Paris, PUF, 1963, p. 148. Sur ce point voir mon étude «L'art de la nature, Sur la possibilité d'une expérience esthétique de la nature », dans Mikel Dufrenne et l'esthétique, entre phénoménologie et philosophie de la nature (dir. J-.B Dussert et A. Jdey), Rennes, PUR, 2016.
} 
prendre la figure terrifiante d'une altérité irréductible qui crée l'effroi et le choc. Éprouver une émotion esthétique, et « vivre en elle, c'est bien prendre attitude, évaluer esthétiquement $»^{1}$.

On peut conclure avec le mot de Baudelaire :

Oui, l'imagination fait le paysage, il faut que tout cela devienne tableau par le moyen de l'impression poétique rappelée à volonté ${ }^{2}$.

Il ne suffit pas d'ouvrir la fenêtre pour saisir un paysage et la nature seule ne nous propose qu'un assemblage divers de réalités. Le paysage pour Baudelaire n'apparaît que « par moi, par ma grâce propre, par l'idée ou le sentiment que j'y attache ${ }^{3}{ }^{3}$. L'expérience esthétique du paysage, et non pas une simple perception naturaliste, suppose l'intervention active, mobile, de l'imagination qui s'empare du donné, le compose et le dispose en tableau; une imagination qui sait jouer et rejouer «à volonté » le jeu de la meilleure disposition, du meilleur angle, jusqu'à atteindre une intuition vive et dense, riche d'émotions - soit une imagination perceptive, c'est-à-dire une vision où «l'effectivement réel fait fonction de figuration » ${ }^{4}$. Ainsi une expérience esthétique de la nature est possible qui, sans nécessairement requérir une mise entre parenthèses des positions d'existence, repose néanmoins sur un jeu intentionnel où l'imagination tient la première place qui entrelace les modes d'apparition et nous convie à un échange dynamique entre le réel, l'irréel, le comme si et le fictum.

\section{Bibliographie}

Aristote, La Poétique, Paris, Seuil, 1980.

Baudelaire, «Salon de 1859 », article «Paysage », dans Baudelaire, Critique d'art, Paris, Folio, 1992.

Carlson A., Nature \& Landscape. An introduction to environmental Aesthetics, New York: Columbia University Press, 2009.

Crowther P., The Kantian Aesthetics. From Knowledge to the Avant-Garde, Oxford, Oxford University Press, 2010.

\footnotetext{
${ }^{1}$ Hua XXIII, p. 376 [389].

${ }^{2}$ Baudelaire, «Salon de 1859 », article « Paysage », dans Baudelaire, Critique d'art, Paris, Folio, 1992, p. 325.

${ }^{3}$ Ibid.

${ }^{4}$ Hua XXIII, p. 488 [517].
} 
Dufrenne M., Le Poétique, Paris, PUF, 1963.

Husserl E., Husserliana XXIII, trad. fr. : Phantasia, Conscience d'image, Souvenir, Grenoble, Jérôme Millon, 2002.

Husserl E., Lettre à Hofmannsthal, du 12 juillet 1907, dans La Part de l'œil, 1991, Bruxelles, trad. É. Escoubas, p. 13-15.

Ingarden R., "Le tableau, l'image picturale», dans Esthétique et ontologie de l'œuvre d'art, Choix de textes 1937-1969 (trad. fr. P. Limido), Paris, Vrin, 2011.

Kant E., Critique de la faculté de juger, Paris, Vrin, 1984.

Limido P., «L'art de la nature, Sur la possibilité d'une expérience esthétique de la nature », dans J.-B. Dussert et A. Jdey (dir.), Mikel Dufrenne et l'esthétique, entre phénoménologie et philosophie de la nature, Rennes, PUR, 2016, p. 241260.

Lories D., «Remarks on Aesthetic Intentionality: Husserl or Kant», International Journal of Philosophical Studies, vol. 14 (1), 2006, p. 31-49.

Lyotard J.-F., «Scapeland», Revue des sciences humaines, tome 30, n² 209, Janmars 1988, p. 39-48.

Margolis J., «La spécificité ontologique des œuvres d'art» dans D. Lories (dir.), Philosophie analytique et esthétique, Paris Klincksieck, 2004, p. 211-219.

Roger A., Court traité du paysage, Paris, Gallimard, 1997.

Stolnitz J., «L'attitude esthétique », dans D. Lories (dir.), Philosophie analytique et esthétique, Paris, Klincksieck, 2004, p. 103-114. 